\title{
Equilibrium polyelectrolyte bundles with different multivalent counterion concentrations
}

\author{
Mehmet Sayar \\ College of Engineering, Koc University, Istanbul, Turkey
}

Christian Holm

Institut für Computerphysik, Universität Stuttgart, Pfaffenwaldring 27, 70569 Stuttgart, Germany

(Received 12 May 2010; revised manuscript received 29 July 2010; published 2 September 2010)

\begin{abstract}
We present the results of molecular-dynamics simulations on the salt concentration dependence of the formation of polyelectrolyte bundles in thermodynamic equilibrium. Extending our results on salt-free systems we investigate here deficiency or excess of trivalent counterions in solution. Our results reveal that the trivalent counterion concentration significantly alters the bundle size and size distribution. The onset of bundle formation takes place at earlier Bjerrum length values with increasing trivalent counterion concentration. For the cases of $80 \%, 95 \%$, and $100 \%$ charge compensation via trivalent counterions, the net charge of the bundles decreases with increasing size. We suggest that competition among two different mechanisms, counterion condensation and merger of bundles, leads to a nonmonotonic change in line-charge density with increasing Bjerrum length. The investigated case of having an abundance of trivalent counterions by $200 \%$ prohibits such a behavior. In this case, we find that the difference in effective line-charge density of different size bundles diminishes. In fact, the system displays an isoelectric point, where all bundles become charge neutral.
\end{abstract}

DOI: 10.1103/PhysRevE.82.031901

PACS number(s): 87.15.-v, 36.20.Ey, 82.35.Rs, 33.15.Bh

\section{INTRODUCTION}

Semiflexible charged biopolymers such as F-actin, microtubules, or DNA aggregate into bundles in the presence of multivalent counterions. Experimental results suggest that, even though these bundles have a broad length distribution, their lateral dimensions (i.e., bundle diameter) are rather well controlled [1-7]. Theoretical explanations for the finite size of the bundles have been based on assembly defects in toroidal aggregates [8], on kinetic limitations [9-16], on chirality of the aggregates [17], or on thermodynamic equilibrium via a balance of competing interactions $[18,19]$.

The origin of the attraction among such highly charged molecules is short-range ionic correlations of multivalent counterions, a phenomenon that cannot be explained on the level of mean-field theories such as the Poisson-Boltzmann $[11,20-23]$. For biomolecules such as DNA, F-actin, or microtubules this correlation induced attraction together with the stiffness of the molecules favors bundle formation $[1,5,7,24]$.

In our earlier work [18] we have shown that by controlling the dielectric constant of the solution (and thereby tuning the Bjerrum length) one can create a delicate balance among the electrostatic forces (which favor aggregation) and entropy (which oppose bundle growth) and stabilize finitesize aggregates in thermodynamic equilibrium. We have also observed that a key parameter in determining the aggregate size and size distribution is the concentration of the trivalent counterions, which act as the gluing agent in this system. In our earlier study, the concentration of the trivalent counterions was just sufficient to neutralize the system. Depletion of the free trivalent ions from solution leads to the termination of the bundle growth as well.

In the present work we investigate bundle formation in a system of monodisperse charged semiflexible polymers under different trivalent counterion concentrations. Using molecular-dynamics simulations, we analyze the size and size distribution of polyelectrolyte (PE) bundles under deficiency and abundance of trivalent counterions. By observing the effective charge density of different size aggregates we aim to present a complete analysis of bundle formation in the presence of many-body effects, which has been omitted in many theoretical and computational studies due to its complexity.

\section{MODEL}

The bead-spring model of PE chains and trivalent counterions utilized here is identical to the one used in our earlier study of PE bundles [18]. In this model all particles have identical diameter $\sigma$ and interact via a purely repulsive Lennard-Jones interaction,

$$
U_{L J}(r)= \begin{cases}4 \epsilon_{L J}\left[\left(\frac{\sigma}{r}\right)^{12}-\left(\frac{\sigma}{r}\right)^{6}+0.25\right], & r<r_{\text {cut }} \\ 0, & r \geq r_{\text {cut }},\end{cases}
$$

with cutoff distance $r_{c u t}=2^{1 / 6} \sigma$ and interaction strength $\epsilon_{L J}$ $=k_{B} T$. The PEs are monodisperse, where each chain is composed of 30 negatively charged beads connected via finitely extensible nonlinear elastic (FENE) bonds,

$$
U_{F}(r)=-\frac{1}{2} k_{F} R_{F}^{2} \ln \left[1-\left(\frac{r}{R_{F}}\right)^{2}\right],
$$

for $r<R_{F}$, with a spring constant of $k_{F}=7 k_{B} T$ and cutoff distance $R_{F} / \sigma=2$. The PEs are semiflexible with a harmonic angular potential

$$
U_{\theta}(r)=\frac{1}{2} k_{\theta} \theta^{2}
$$

of stiffness $k_{\theta}=100 k_{B} T$, where $\theta$ is the angle between any three consecutive beads along the PE chain.

The Coulomb interactions, 


$$
U_{C}\left(r_{i j}\right)=\lambda_{B} k_{B} T \frac{q_{i} q_{j}}{r_{i j}},
$$

are calculated using a version of the particle-particleparticle-mesh (P3M) method [25]. The Bjerrum length $\lambda_{B}$ is defined as the distance at which the energy between two particles with unit charges in a given dielectric medium is exactly equal to the thermal energy, $k_{B} T$. The Bjerrum length is used as the control parameter for the strength of the electrostatic interactions.

All four systems studied here contain 61 PEs with different trivalent counterion concentrations. For the $100 \%$ trivalent counterion case the total charge of the PEs $(61 \times 30=1830)$ is compensated by 610 trivalent counterions. For the $80 \%$ and $95 \%$ trivalent counterion concentration systems, only $80 \%$ and $95 \%$ of the total charge of the PEs are compensated by trivalent counterions, respectively. The system is neutralized by adding sufficient amount of monovalent counterions in both cases. For the $200 \%$ trivalent counterion concentration case a total of 1220 trivalent counterions are added to the 61 PEs. This system is neutralized by adding monovalent coions.

The equilibrium configurations for the $100 \%$ counterion concentration have been obtained in our previous study [18]. The $80 \%, 95 \%$, and $200 \%$ trivalent counterion concentration simulations are all started from a randomly chosen equilibrium snapshot from the $100 \%$ counterion system. For the $80 \%$ and $95 \%$ counterion concentrations sufficient number of trivalent counterions from the $100 \%$ system is randomly replaced by monovalent counterions. In order to keep the total charge neutral, extra monovalent counterions are added at randomly chosen coordinates within the simulation box. If a randomly chosen coordinate for an inserted monovalent counterion violates the excluded volume of any of the already present particles, a new location is chosen. For the $200 \%$ counterion concentration case extra trivalent counterions and monovalent coions are added to the system at random coordinates, as described for the other two concentrations. All starting conformations are energy minimized after the addition of the coions and counterions.

The PE monomer number density is fixed for all simulations as $\rho / \sigma^{-3}=7.5 \times 10^{-5}$, which is dilute enough to allow us to ignore bundle-bundle contacts in the analysis of the simulation trajectories. The simulations are performed in a cubic box with periodic boundary conditions.

Parallel tempering (PT) method [26,27] with Bjerrum length as the tempering parameter is used to sample the phase space of these systems. In each case, the ensemble consists of 49 replicas with different Bjerrum length values covering the range $\lambda_{B} / \sigma=1.50-2.19$. For the simulation of individual replicas hybrid Monte Carlo (HMC) method $[28,29]$ is used, where trial configurations for each Monte Carlo move are generated by molecular dynamics (MD). MD simulations are performed with the ESPResSo package [30]. A time step of $\Delta t=0.005 \tau$ is used, where $\tau$ is the usual Lennard-Jones time unit. We integrated for $50 \tau$ for each HMC trial, which yields a good combination of acceptance ratio and efficient sampling of the configurational space. For each PT exchange attempt, we have done nine HMC moves, which are called a cycle. The ensemble of replicas has been equilibrated for up to 300 cycles, and data are collected from an additional run of at least 200 cycles, which took roughly three CPU years on Intel Xeon processors $(2.4 \mathrm{GHz})$. The equilibrium aggregate size and size distribution have been monitored and used as the convergence criteria for our simulations.

In the following analysis a trivalent counterion within $3 \sigma$ distance of a PE rod is assumed to be a condensed counterion. Any two PE rods that share a condensed trivalent counterion are assumed to be in the same bundle. The aggregate size and size distribution results do not depend on the choice of the condensation distance criteria. The effective linecharge-density results also remain qualitatively identical if the analysis is performed with different distance criteria. Monovalent counterions remain in solution dominantly and they do not contribute to the reduction of the effective linecharge density of PE rods and formation of bundles.

\section{RESULTS AND DISCUSSION}

Attraction among like charged polyelectrolytes can be induced by introducing multivalent counterions into the solution [11,21-23]. In the case of rodlike molecules this attraction leads to the aggregation of polyelectrolytes into bundlelike structures. In our previous study [18] we have shown that in a narrow window of Bjerrum length values finite-size bundles can be observed in thermodynamic equilibrium. In this earlier work, a system of $61 \mathrm{PE}$ rods of length 30 beads each was neutralized by trivalent counterions only. In the following, this system will be referred to as the $100 \%$ case. In this study, we will explore the additional cases of different neutralization degrees with trivalent counterions, namely, the cases of $80 \%, 95 \%$, and $200 \%$, and compare them with the $100 \%$ case.

The concentration of trivalent counterions dramatically changes the aggregation behavior of PE rods as seen in Fig. 1. For all four concentrations representative equilibrium snapshots are taken at Bjerrum length $\lambda_{B} / \sigma=1.95$, which is in the narrow window where finite-size bundles are seen for the $100 \%$ case [18] (Fig. 1, bottom left). For the $100 \%$ case several bundles of different size are observed simultaneously in the solution. Even though most of the trivalent counterions are condensed on to the PE rods (white beads attached to the PE rods), some are still free (red beads dispersed in the simulation box). The dominant mode of merging for PE rods is observed to be the zipper model [9,16,31]: an isolated PE rod approaches a preexisting bundle at a $90^{\circ}$ angle and slides until the tip of the PE rod is close to the tip of the bundle. At this point the PE rod rotates to become parallel with the bundle axis, which is followed by sliding of the PE rod until it aligns with the rest of the rods in the bundle. When the trivalent counterion concentration is reduced to 95\% (Fig. 1, top right), one can still observe different size bundles in solution. However, at $80 \%$ for $\lambda_{B} / \sigma=1.95$ all bundles, except for small two to three molecule ones, are destroyed (Fig. 1, top left). For both $80 \%$ and $95 \%$ at $\lambda_{B} / \sigma=1.95$ the monovalent counterions (not shown in Fig. 1 for visual clarity) do not condense onto the PE rods but mostly remain in solution. 


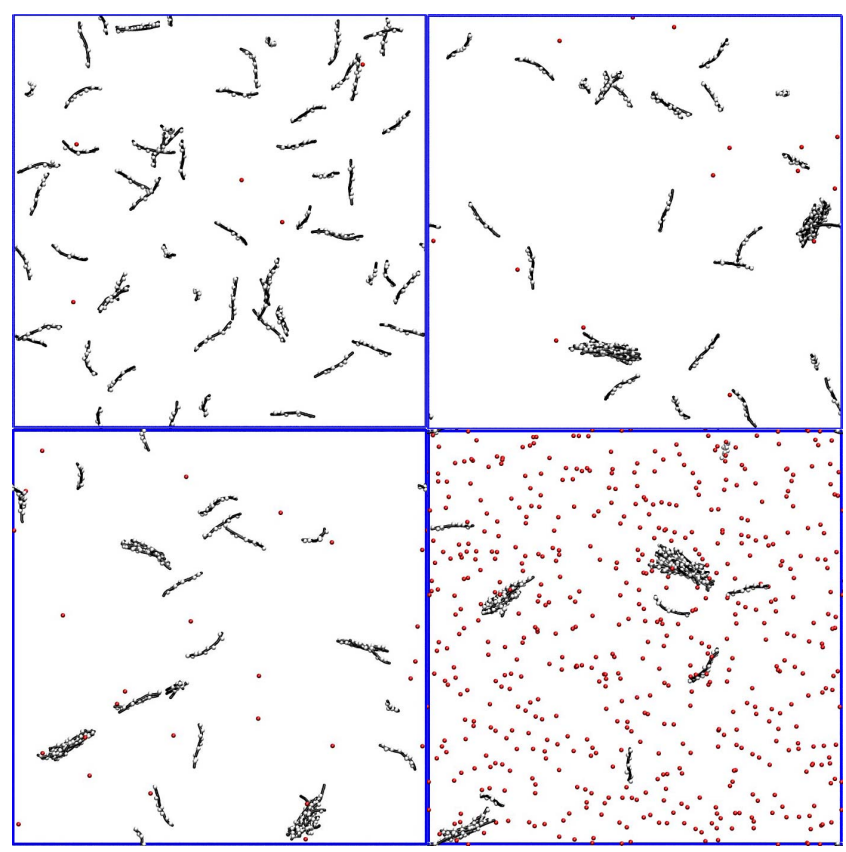

FIG. 1. (Color online) Snapshots from systems with $80 \%$ (top left), $95 \%$ (top right), $100 \%$ (bottom left), and 200\% (bottom right) trivalent counterion concentrations at $\lambda_{B} / \sigma=1.95$. PE rods are shown as black tubes. Condensed (beads attached to the PE rods) and free (isolated beads) trivalent counterions are shown as white and red (gray) spheres, respectively. Monovalent counterions (for $80 \%$ and $95 \%$ cases) and coions (for $200 \%$ case) are not shown for visual clarity.

Upon increasing the trivalent counterion concentration to $200 \%$ the aggregate size distribution changes once again, such that the system is composed of a few large bundles (Fig. 1 , bottom right). The monovalent coions (also not shown for visual clarity) remain in solution similar to the monovalent counterions.

In order to understand how the aggregate size distribution changes as a function of Bjerrum length, we have analyzed the equilibrium distribution of bundles for the range $\lambda_{B} / \sigma$ $=1.5-2.2$. As we observed in our earlier study (also seen in Fig. 2), for the $100 \%$ case, the equilibrium aggregate size monotonically increases with increasing Bjerrum length. As the average aggregate size increases, the aggregate polydispersity shows a nonmonotonic dependence on $\lambda_{B}$ (Fig. 3). In the interval $\lambda_{B} / \sigma=1.80-1.95$ the polydispersity of the bundles monotonically increases. Several finite-size bundles are observed, where the PE rods continuously merge with or split up from these bundles, displaying a dynamic equilibrium (see Fig. 5 for the probability distribution of PEs of different size bundles and Fig. 1 for snapshots from the system). Beyond $\lambda_{B} / \sigma>1.95$ the bundle polydispersity starts decreasing since the bigger aggregates start growing further at the expense of the smaller ones. Eventually, the system is dominated by a few aggregates with sizes larger than 20 and considering that there are only 61 rods in total, the simulation results become unreliable.

Compared to the $100 \%$ case, the most dramatic change is observed for the $80 \%$ case, where within the $\lambda_{B}$ range covered in this study, both the average aggregate size (Fig. 2)

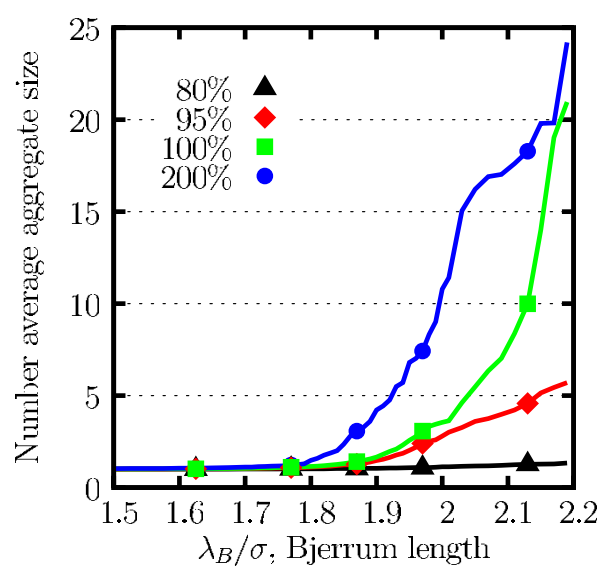

FIG. 2. (Color online) Number average aggregate size for four different trivalent counterion fractions. The aggregate size is calculated as $N_{n}=\sum N_{i} f_{i}$, where $f_{i}$ is the fraction of aggregates of size $N_{i}$. Here and in Figs. 3, 4, and 6 the symbols on the curves are shown for every fifth data point to improve visibility.

and aggregate polydispersity (Fig. 3) only slightly increase above 1 . The aggregation of the rods is limited to two and to a small extent three molecule bundles. Since the system is neutral, at a sufficiently high $\lambda_{B}$ value all the rods should merge into a single bundle by attracting both monovalent and trivalent counterions. However, as seen in Figs. 2 and 3 the electrostatic attraction at the highest $\lambda_{B}$ value used in this study is still insufficient to form a single aggregate with all of the PEs. More interestingly, no phase separation is observed in the system. In other words, formation of a larger bundle via depletion of trivalent counterions from the remaining dispersed rods is not observed for the $80 \%$ case.

The potential of mean force (PMF) can be used to understand the nature of interactions among PE rods at different Bjerrum length values. In Fig. 4 PMF curves at different electrostatic strengths are shown. PMF curves are obtained by inverting the radial distribution function for the center of mass of the PE chains. For the $80 \%$ case for $\lambda_{B} / \sigma \leq 1.6 \mathrm{PE}$ rods display purely repulsive interactions. With increasing $\lambda_{B}$

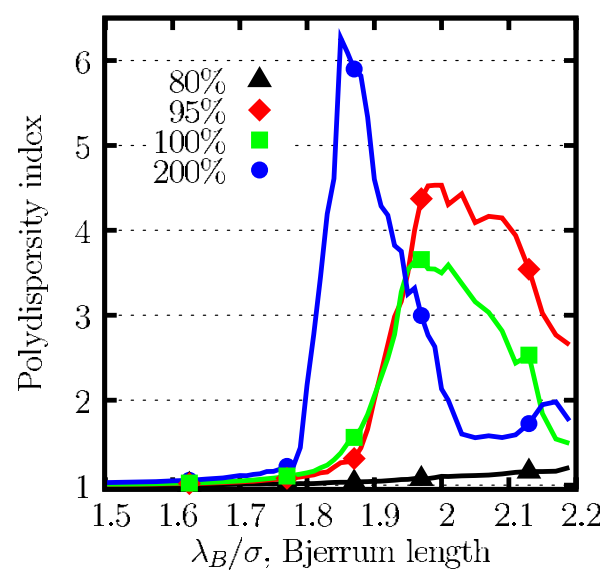

FIG. 3. (Color online) Polydispersity index for different trivalent salt concentrations. The polydispersity index is calculated as the ratio of weighted average $\left(N_{w}=\sum N_{i}^{2} f_{i} / \sum N_{i} f_{i}\right)$ to number average $\left(N_{n}\right)$ aggregate size. 

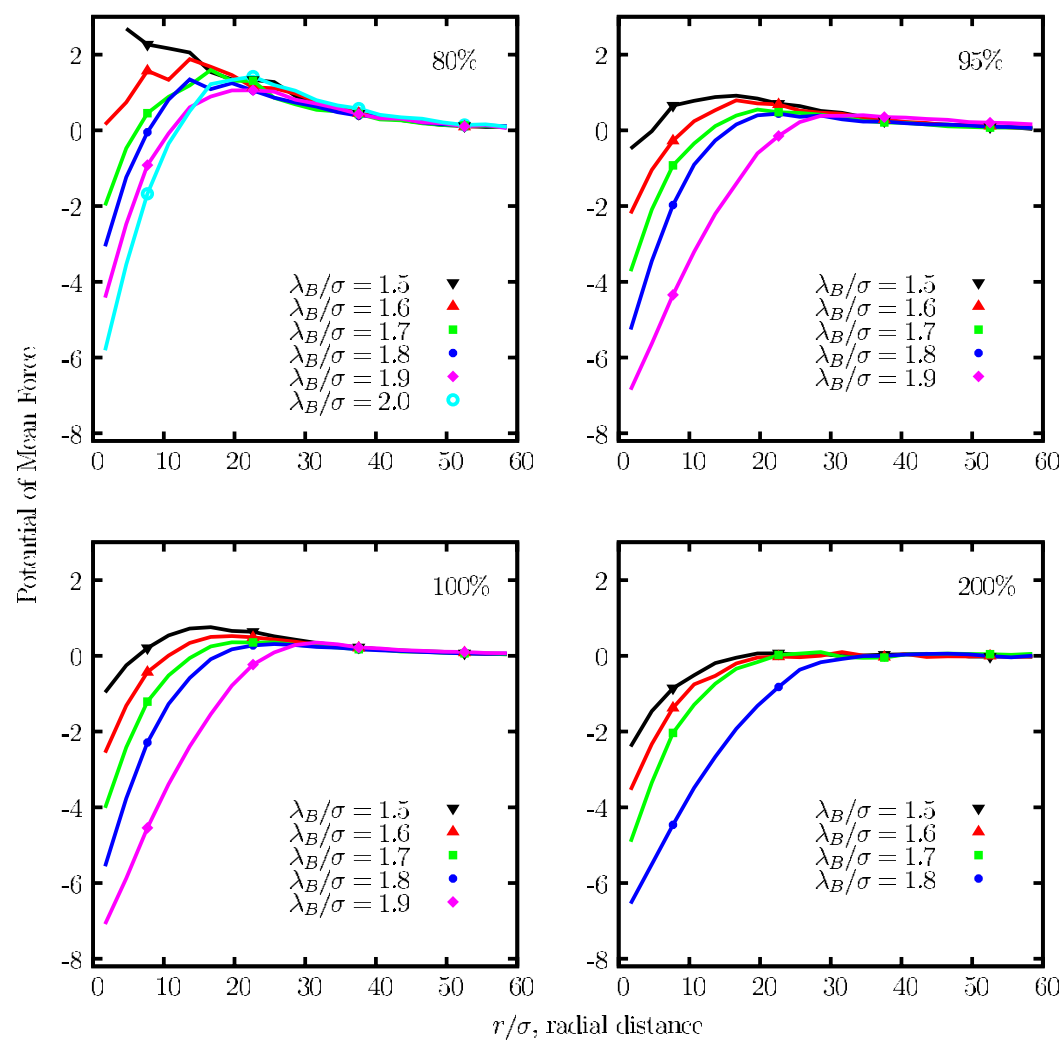

FIG. 4. (Color online) The potential of mean force for the $80 \%$ (top left), 95\% (top right), $100 \%$ (bottom left), and $200 \%$ (bottom right) cases obtained by inverting the radial distribution function of the center of mass of the PE chains. the PMF becomes attractive at short distances, however, the presence of an energy barrier and a long-range repulsive tail in the PMF prevents the formation of large size aggregates for the $80 \%$ case, as discussed above.

For the $95 \%$ case replacement of just $5 \%$ of the trivalent counterions by monovalent ones leads to a dramatic reduction in the average aggregate size compared to the $100 \%$ case (Fig. 2). Up to $\lambda_{B} / \sigma=1.90$ the curves for the $95 \%$ and $100 \%$ cases remain almost identical. However, once the formation of large size bundles starts (beyond $\lambda_{B} / \sigma=1.90$ ) aggregation in $95 \%$ case lags behind. Unlike the $80 \%$ case, here the concentration of trivalent counterions is sufficient for the growth of large size bundles. Comparison of the distribution of PEs into different size aggregates (Fig. 5) reveals an interesting difference between $100 \%$ and $95 \%$ cases: in the $95 \%$ case the formation of large bundles leads to an extinction of medium-sized aggregates, while single rods and two to three molecule bundles still exist in the system at $\lambda_{B} / \sigma=2.00$. As will be discussed below, this is associated with the insufficient number of trivalent counterions in the system. The number of trivalent counterions is not large enough to maintain the medium and large size bundles simultaneously. This suggests the formation of a two phase system: one phase consists of large size (however still finite) bundles, which attracts a higher fraction of trivalent counterions per PE rod. The second phase consists of single rods or two to three molecule bundles, where the trivalent counterion concentration per PE rod is less than the first phase, which will be discussed further when we compare the effective line-charge density of different size bundles.

Comparison of the PMF curves for the 100\% and 95\% cases shows that at low $\lambda_{B}$ even though the energy barrier is still present, its value is highly reduced compared to the $80 \%$ case. With increasing $\lambda_{B}$ the energy barrier disappears, which coincides with the sharp increase in average aggregate size for $95 \%$ and $100 \%$ cases. Above $\lambda_{B} / \sigma \geq 1.90$ the repulsive tail of the PMF curve continues to expand, such that beyond $\lambda_{B} / \sigma \geq 2.00$ the PMF curves do not drop to zero at half the box size. This stems from the fact that the radial distribution function used to obtain the PMF curve fails to reach the bulk density (results not shown), which is a direct consequence of the finite size of the system (only 61 rods).

For all three concentrations considered up to here, the size and distribution of the finite-size bundles are directly associated with the insufficient number of trivalent counterions. On the contrary, for the $200 \%$ case the system has an abundance of trivalent counterions, as seen in Fig. 2. In this case the onset of aggregation and the growth of the finite-size bundles take place at lower Bjerrum length values compared to other concentrations. In general, the higher the the trivalent salt concentration is, the earlier the onset of aggregation sets in. Furthermore, the overall bundle size at fixed Bjerrum length is always the highest in the $200 \%$ case when compared with other salt concentrations. Looking at the polydispersity index in Fig. 3 we also note a sharper increase of the polydispersity index with increasing Bjerrum length compared to the other three cases. Note that for the $200 \%$ case no energy barriers are seen in the PMF curves (Fig. 4). This phenomenon is directly related with the net charge of the aggregates, which will be discussed further below.

As seen in Fig. 5, at $\lambda_{B} / \sigma=1.75$ for all salt concentrations the majority of the aggregates are dispersed single rods. Even at such a low $\lambda_{B}$ there seems to be more bundle nucleation sites for the $200 \%$ trivalent counterion case, so that already a finite fraction of the PEs are in two and three molecule bundles. Upon increasing the Bjerrum length we first 

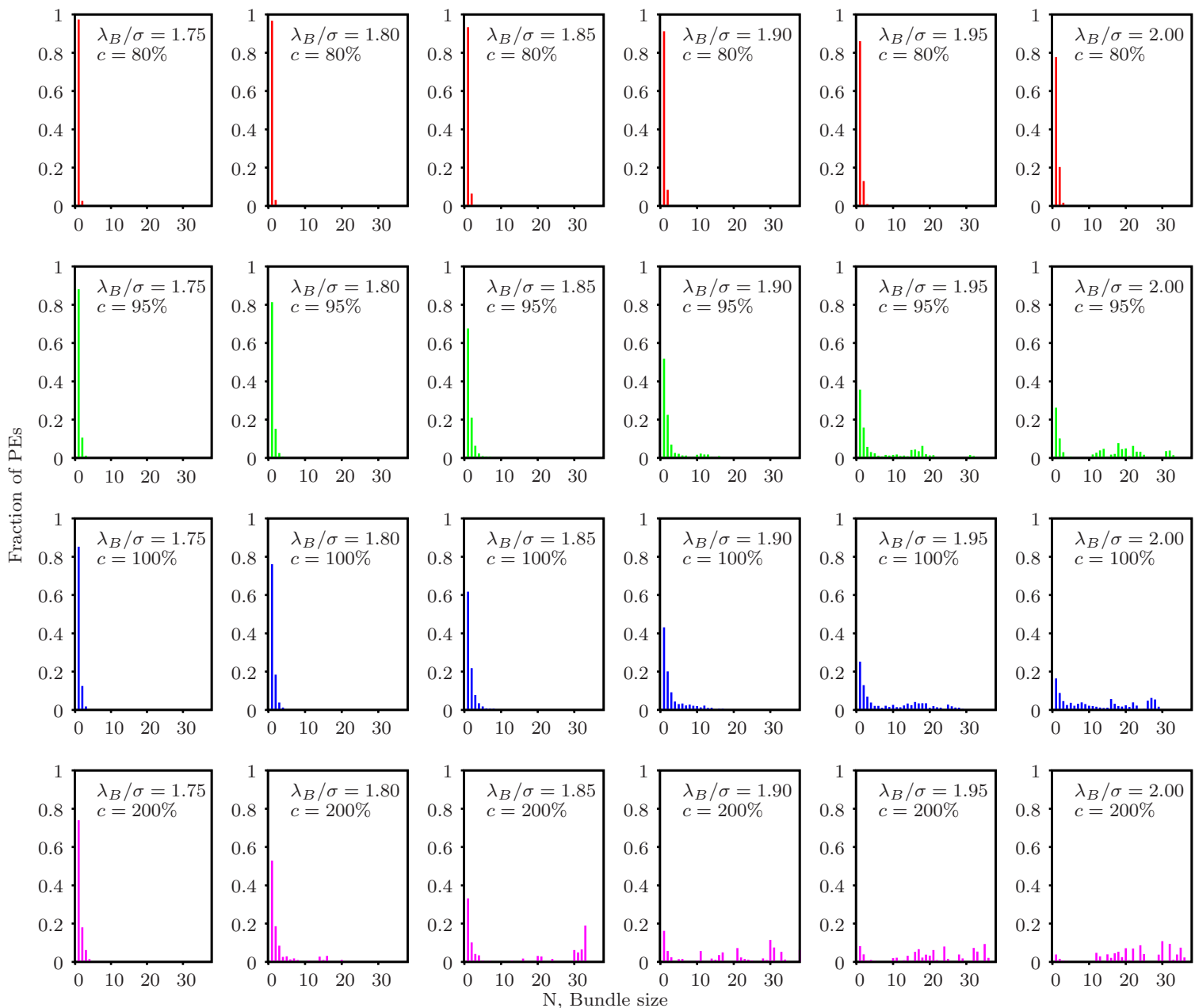

FIG. 5. (Color online) Probability of observing a PE rod in a bundle of size $N$ as a function of the Bjerrum length values of $\lambda_{B} / \sigma=1.75,1.80,1.85,1.90,1.95$, and 2.00 at four different salt concentrations, $80 \%, 95 \%, 100 \%$, and $200 \%$.

see that bigger bundles appear for the $200 \%$ salt case at around $\lambda_{B} / \sigma=1.80$. In a very narrow window of Bjerrum lengths up to 1.85 we can see distributions of aggregates, which seem to indicate that the single rods are in equilibrium with a phase that contains finite-size aggregates. At higher Bjerrum lengths for the $200 \%$ case, the situation is not so clear since the distribution of aggregates becomes flat, and larger bundles appear.

For a better understanding of the formation of bundles, we also calculated the effective line-charge density $\left(f_{e}\right)$ of the PE rods (Fig. 6). For a PE rod in a bundle, $f_{e}$ is obtained by dividing the net charge of the bundle, after taking into ac-
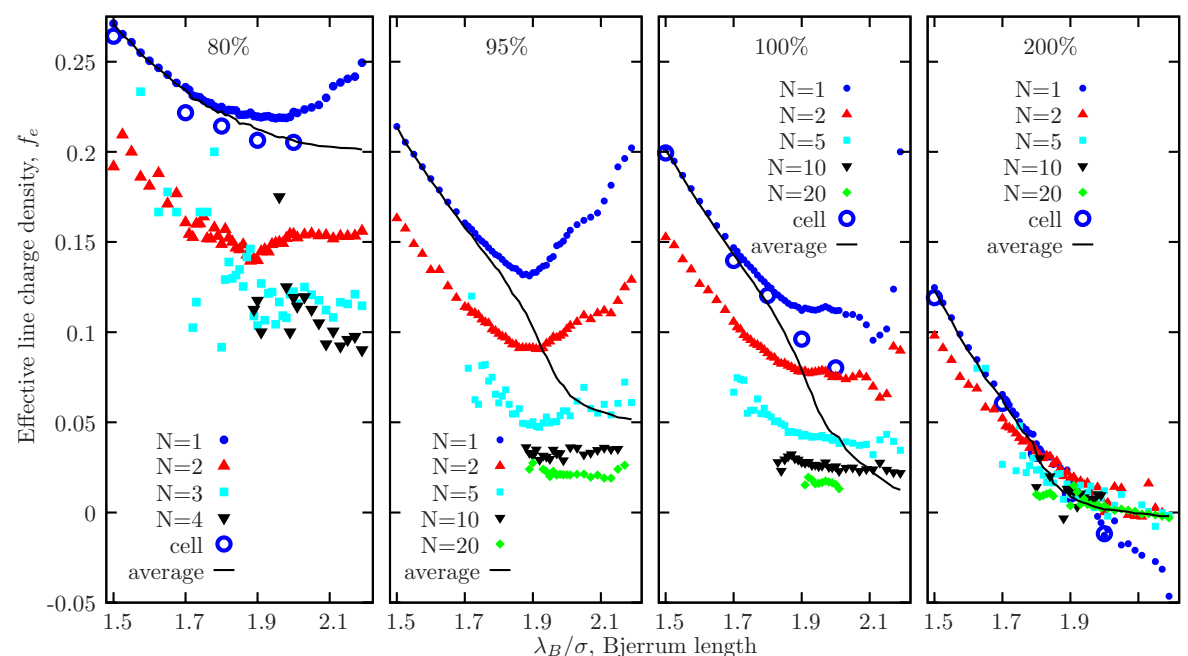

FIG. 6. (Color online) The effective line-charge density $\left(f_{e}\right)$ for different size bundles $(N)$ as a function of the Bjerrum length. The results are shown for $80 \%$, $95 \%, 100 \%$, and $200 \%$ trivalent counterion concentration cases. The open circles are obtained from simulations of a single PE rod in a cell with the same concentration of counterions and illustrate the behavior of $f_{e}$ in the absence of many-body interactions. 
count the condensed trivalent counterions, by the length of PEs and the total number of PEs in the bundle. Any trivalent counterion within $3 \sigma$ distance of a PE bead is considered to be condensed. For entropic reasons monovalent counterions do not make a significant contribution to the effective line charge and therefore they are excluded from the calculation (results not shown).

In Fig. 6 the solid lines, labeled as "average," represent the average effective line-charge density for the whole system, regardless of the aggregation state of the PE rods. For all four cases the average $f_{e}$ decreases with increasing Bjerrum length. For the $80 \%$ case $f_{e}$ saturates at $f_{e}=0.20$. This is the lowest $f_{e}$ value that the system can attain since no more free trivalent counterions are left in solution. As seen in the aggregation results the $80 \%$ case is dominated by the inadequate supply of trivalent counterions. For the $95 \%$ case the $f_{e}=0.05$ limit is also reached at the highest Bjerrum length considered here, but for the $100 \%$ case the lowest limit $\left(f_{e}=0.0\right)$ is still not reached and there have to be free trivalent counterions in solution even at $\lambda_{B} / \sigma=2.19$.

For the $95 \%$ and $100 \%$ cases the average effective linecharge density initially decreases linearly, with a slope of $m \approx-0.30$. Around $\lambda_{B} / \sigma=1.90$ the slope of the $f_{e}$ curve increases further to a value of $m \approx-0.45$ and decreases once again beyond $\lambda_{B} / \sigma=1.95$. The change in slope takes place roughly where we first observe formation of bundles in $95 \%$ and $100 \%$ cases as seen in Fig. 2. In other words aggregation of bundles enhances condensation rate of trivalent counterions within a narrow window prior to the system reaching the saturation limit. For the $200 \%$ case this enhanced condensation window is much narrower. As we have seen in Figs. 2 and 3, the aggregation takes place much more rapidly for this case. Hence, one cannot observe the increase in slope as clearly as the $95 \%$ and $100 \%$ cases.

Comparison of these bulk simulation results with simulations of a single PE rod and counterions isolated in a cell, where many-body interactions are prohibited, also supports the idea that the change in slope is due to the aggregation enhanced condensation. The results from cell simulations for $100 \%$ and $200 \%$ cases, shown with open circles in Fig. 6, display a constant slope for the whole $\lambda_{B}$ region investigated. The slope matches the bulk behavior prior to aggregation $(m \approx-0.30)$ even at high $\lambda_{B}$. Since there is only a single rod in the cell, aggregation is prohibited and therefore a change in slope of $f_{e}$ is also not observed. Cell simulation results for $80 \%$ case once again behave differently due to the inadequate supply of trivalent counterions.

The difference between the cell and bulk simulation results is associated with how PE rods reduce their electrostatic energy. PE rods have two different mechanisms for lowering their electrostatic energy as the strength of the electrostatic interactions increases with increasing $\lambda_{B}$. The first mechanism is via counterion condensation, which is the only mechanism available in cell simulations. This mechanism is also dominant at low $\lambda_{B}$ values in bulk simulations. The second mechanism for lowering the electrostatic energy, which can be seen only in a bulk simulation of PE rods, is via the merger of PE rods (or smaller bundles) into larger bundles. This second mechanism becomes dominant at high $\lambda_{B}$ values. In the bulk system the interplay and competition of these two different mechanisms lead to the observed nonmonotonic behavior for $f_{e}$.

In order to understand the interplay of these two different mechanism one can look at the effective line-charge density, $f_{e}$, for bundles of different sizes in bulk simulation. In Fig. 6 $f_{e}$ for aggregates of sizes $N=1,2,5,10$, and 20 are shown. For any given size $N$, data points exist only if such a bundle is observed at the given Bjerrum length. For example, for the $95 \%$ case the data points for $N=5$ are seen only above $\lambda_{B} / \sigma=1.70$ because below this value aggregates of size $N=5$ are not observed. For the $80 \%$ case, since no aggregates larger than $N=4$ molecules exist, data are shown for $N=1,2$, 3 , and 4 . The $f_{e}$ values shown in the plot are averaged over all observations of a given aggregate size at a $\lambda_{B}$ value. The error in $f_{e}$ increases if that aggregate size is seen very rarely at that $\lambda_{B}$ value. For example, the highly scattered distribution of $f_{e}$ values for $N=3$ and 4 molecule bundles in $80 \%$ case is due to the low probability for such bundles to occur.

For concentrations of $80 \%, 95 \%$, and $100 \%$, single rods and to some extent other small size bundles initially display a linearly decreasing $f_{e}$ with increasing $\lambda_{B}$. However, beyond a certain threshold value, the rate of reduction in $f_{e}$ starts to slow down. One can even observe an increase in $f_{e}$ with increasing $\lambda_{B}$, as seen for $N=1$ and 2 for $80 \%$ and $95 \%$ cases. This unexpected rise and/or convergence in $f_{e}$ with increasing electrostatic strength signals the onset of the second mechanism (merger of PE rods) for lowering the electrostatic energy. As the reduction in $f_{e}$ for single rods and small bundles slows down, one observes the emergence of moderate size bundles with lower $f_{e}$ in the solution. Gradually, with increasing $\lambda_{B}, f_{e}$ for these moderate size bundles also saturates and one observes the formation of even larger size aggregates in solution.

At any given $\lambda_{B}$ the larger the bundle size is the lower the $f_{e}$ is. The large bundles attain low $f_{e}$ with two possible pathways. First, after the formation of the bundle the large electric field of the bundle attracts more counterions compared to smaller bundles. Second, the larger bundles form with the merger of smaller size ones, which have already reduced their $f_{e}$ below the average for their own bundle size. The elimination of these low $f_{e}$ bundles from the distribution leads to an increase in the average $f_{e}$ for small size bundles; hence we observe a rise in $f_{e}$.

For the $200 \%$ case the abundance of free trivalent counterions in solution dramatically changes this behavior. At such a high concentration of trivalent counterions difference in $f_{e}$ for different size bundles diminishes. In fact, at $\lambda_{B} / \sigma \approx 1.95$ bundles of all sizes become almost neutral. Upon further increase in $\lambda_{B}$ one observes charge reversal for single rods, which have a higher surface area per rod. For the larger bundles $f_{e}$ fluctuates around zero beyond the isoelectric point.

\section{SUMMARY AND CONCLUSIONS}

In this study, we systematically expanded our earlier work on aggregation of semiflexible polyelectrolytes to different salt concentrations. By changing the trivalent salt concentrations to $80 \%$ and $95 \%$ of the total charge of PE rods we have 
analyzed how the system behaves under a deficiency of the binding trivalent counterions. The $200 \%$ case mimics a solution with an abundance of trivalent counterions.

Our analysis reveals that the aggregate size and size distribution are determined by the trivalent counterion concentration. The higher the trivalent counterion concentration is, the lower the Bjerrum length value for the onset of aggregation. Similar to the $100 \%$ case, for $95 \%$ and $200 \%$ cases the onset of aggregation leads to a sharp increase in both aggregate size and size distribution. However, in all cases the finite-size aggregates remain thermodynamically stable in a narrow window of Bjerrum length values.

For a solution of PE rods and counterions in the absence of an explicit attractive hydrophobic interaction both the attractive and repulsive forces originate from electrostatic interactions. The concentration of trivalent counterions, which act as the glue for PE rods, strictly determines the solution properties.

The effective line-charge density of different size aggregates reveals the role of many-body interactions. Within the cell model, where the system consists of a single PE rod and counterions, one observes a linear monotonic reduction in the effective charge of the PE rod due to counterion condensation. In a solution in addition to counterion condensation, the PE rods can also merge to further lower their electrostatic self-energy. The formation of aggregates increases the rate of condensation for trivalent counterions with increasing Bjerrum length. However, reduction of the self-energy of larger PE bundles comes at the cost of an increase of the electrostatic self-energy of smaller ones in solution.

On the other hand, when the system has an abundance of trivalent counterions, aggregates of all sizes can reduce their electrostatic energy simultaneously. The system also displays an isoelectric point where all aggregates become neutral. Interestingly, beyond the isoelectric point, the remaining single rods in solution display overcharging by condensing more counterions than necessary to compensate their charge. However, the larger size bundles maintain a neutral structure.

\section{ACKNOWLEDGMENTS}

M.S. gratefully acknowledges funding through MaxPlanck-Society Partnership Program, TUBITAK Career Award Grant No. 106T575, and EC support through Marie Curie Intra-European Foundation (Grant No. MCIEF 500604). We also acknowledge additional funding from the DFG (Grants No. SFB 625 and No. Ho1108/11-3). We thank R. Golestanian for scientific discussions.
[1] G. H. Lai, R. Coridan, O. V. Zribi, R. Golestanian, and G. C. L. Wong, Phys. Rev. Lett. 98, 187802 (2007).

[2] L. Haviv, N. Gov, Y. Ideses, and A. Bernheim-Groswasser, Eur. Biophys. J. 37, 447 (2008).

[3] M. Claessens, C. Semmrich, L. Ramos, and A. Bausch, Proc. Natl. Acad. Sci. U.S.A. 105, 8819 (2008).

[4] H. Kwon, Y. Tanaka, A. Kakugo, K. Shikinaka, H. Furukawa, Y. Osada, and J. Gong, Biochemistry 45, 10313 (2006).

[5] J. X. Tang, S. Wong, P. T. Tran, and P. Janmey, Ber. Bunsenges. Phys. Chem. 100, 796 (1996).

[6] J. Käs, H. Strey, J. X. Tang, D. Finger, R. Ezzell, E. Sackmann, and P. A. Janmey, Biophys. J. 70, 609 (1996).

[7] V. Bloomfield, Biopolymers 31, 1471 (1991).

[8] S. Park, D. Harries, and W. Gelbart, Biophys. J. 75, 714 (1998).

[9] H. Fazli and R. Golestanian, Phys. Rev. E 76, 041801 (2007).

[10] B.-Y. Ha and A. J. Liu, Phys. Rev. Lett. 81, 1011 (1998).

[11] Y. Levin, Rep. Prog. Phys. 65, 1577 (2002).

[12] J. F. Stilck, Y. Levin, and J. J. Arenzon, J. Stat. Phys. 106, 287 (2002).

[13] B.-Y. Ha and A. J. Liu, Europhys. Lett. 46, 624 (1999).

[14] M. J. Stevens, Phys. Rev. Lett. 82, 101 (1999).

[15] H. Fazli, S. Mohammadinejad, and R. Golestanian, J. Phys.: Condens. Matter 21, 424111 (2009).

[16] S. Mohammadinejad, H. Fazli, and R. Golestanian, Soft Matter 5, 1522 (2009).
[17] G. M. Grason and R. F. Bruinsma, Phys. Rev. Lett. 99, 098101 (2007).

[18] M. Sayar and C. Holm, EPL 77, 16001 (2007).

[19] M. L. Henle and P. A. Pincus, Phys. Rev. E 71, 060801 (2005).

[20] F. J. Solis and M. O. de la Cruz, Phys. Rev. E 60, 4496 (1999).

[21] C. Holm, P. Kékicheff, and R. Podgornik, Electrostatic Effects in Soft Matter and Biophysics, NATO Science Series IIMathematics, Physics and Chemistry Vol. 46 (Kluwer Academic Publishers, Dordrecht, 2001).

[22] A. Y. Grosberg, T. T. Nguyen, and B. I. Shklovskii, Rev. Mod. Phys. 74, 329 (2002).

[23] H. Boroudjerdi, Y.-W. Kim, A. Naji, R. R. Netz, X. Schlagberger, and A. Serr, Phys. Rep. 416, 129 (2005).

[24] V. A. Bloomfield, Curr. Opin. Struct. Biol. 6, 334 (1996).

[25] M. Deserno and C. Holm, J. Chem. Phys. 109, 7678 (1998).

[26] R. H. Swendsen and J. S. Wang, Phys. Rev. Lett. 57, 2607 (1986).

[27] D. Frenkel and B. Smit, Understanding Molecular Simulation, 2nd ed. (Academic Press, San Diego, 2002).

[28] S. Duane, A. D. Kennedy, B. J. Pendleton, and D. Roweth, Phys. Lett. B 195, 216 (1987).

[29] A. Irback, J. Chem. Phys. 101, 1661 (1994).

[30] H. J. Limbach, A. Arnold, B. A. Mann, and C. Holm, Comput. Phys. Commun. 174, 704 (2006).

[31] T. T. Nguyen and B. I. Shklovskii, Phys. Rev. E 65, 031409 (2002). 